Präv Gesundheitsf 2014 · 9:1-2

DOI 10.1007/s11553-013-0427-9

Online publiziert: 8. Januar 2014

(c) Springer-Verlag Berlin Heidelberg 2013

\title{
Olivia Kada
}

Studiengang Gesundheits- und Pflegemanagement, Fachhochschule Kärnten, Feldkirchen in Kärnten, Österreich

\section{Auf den Spuren von Aaron Antonovsky}

chende Seminare für gesundheitsbewusste Führung sensibilisiert werden können. Es konnte eine Veränderung in der Selbstführung festgestellt werden (v. a. Anstieg der gesundheitsbezogenen Selbstwirksamkeitserwartung). Darüber hinaus stieg die Achtsamkeit der Führungskräfte für die Gesundheit der Mitarbeiter und die Bedeutung gesundheitsförderlicher Arbeitsbedingungen deutlich an.

Ebenfalls mit betrieblicher Gesundheitsförderung befassen sich Kogel et al. Sie stellen die Ergebnisse der Evaluation des Programms „Bewegte Pause“ vor. Die Ergebnisse der quasiexperimentellen Studie sprechen für die Wirksamkeit des Programms v. a. hinsichtlich der Reduktion gesundheitlicher Beschwerden (z. B. Rückenschmerzen).

Hofmann et al. widmen sich in einer systematischen Literaturübersicht der Frage, ob Interventionen schulischer Gesundheitsförderung soziale Ungleichheit weiter vergrößern, indem sie bei Schülern mit unterschiedlichen Merkmalen eine unterschiedlich starke Wirkung entfalten. Eine derartige Verstärkung sozialer Ungleichheit zeigte sich für Ernährungs- und Bewegungsinterventionen, während andere Interventionen durchaus zur Reduktion sozialer Ungleichheit geeignet waren.

Kada beschäftigt sich in ihrem Beitrag mit der Förderung der studentischen Partizipation in der Gesundheitsberichterstattung im Bereich Studierendengesundheit und knüpft damit an Überlegungen zur partizipativen Gesundheitsforschung in der Ausgabe 8(3) dieser Zeitschrift an. Das vorgestellte didaktische Konzept gestaltet nach den Prinzipien Forschenden Lernens zeigt auf, wie Forschung durch die Beteiligung von Studierenden bereichert werden kann und wie
Studierende von der Einbindung in Forschung profitieren können.

Becker et al. richten mit dem Gesundheit-Sport-Biografiesurvey den Fokus auf die Auswirkungen des Gesundheitszustands und körperlicher Beeinträchtigung auf die sportliche Aktivität. Erwartungsgemäß zeigte sich, dass gewisse Erkrankungen mit reduzierter sportlicher Aktivität assoziiert sind. Die Daten können als Ausgangspunkt für die Planung und Umsetzung gesundheitsförderlicher Maßnahmen dienen.

Bucksch und Dreger stellen ein verhaltensepidemiologischs Rahmenkonzept zu sitzenden Verhaltensweisen im Kindes- und Jugendalter vor. Sitzendes Verhalten ist ein eigenständiger, von Bewegungsverhalten unabhängiger Risikofaktor. Der Beitrag zeigt Möglichkeiten zur Reduktion von Sitzzeiten auf sowie Desiderata in diesem neuen Forschungsfeld.

Lischer et al. befassen sich mit der Identifikation vulnerabler Personen im Glücksspielbereich, um eine Basis für primärpräventive Maßnahmen zu schaffen. Im Rahmen der Mixed-Methods-Studie zeigte sich, dass Personen im Alter von 26-45 Jahren sowie Migranten (aufgrund ihrer spezifischen Lebensumstände) besonders häufig von Spielsperren durch Casinos betroffen sind. Primärpräventive Maßnahmen sollten folglich auf diese vulnerablen Gruppen zugeschnitten werden.

Mergenthal stellt in ihrer narrativen Literaturübersicht Möglichkeiten zur Förderung der Gesundheitskompetenz türkischer Migranten/innen vor. Als besonders Erfolg versprechend kristallisierten sich Maßnahmen heraus, die an kulturell akzeptierten Standorten abgehalten werden, sowie Maßnahmen von längerer Dauer. 


\section{Editorial}

Tesky et al. widmen sich der Förderung kognitiv stimulierender Freizeitaktivitäten bei Migranten/innen, einem Schutzfaktor für Demenz. Vorgestellt werden die Ergebnisse einer Pilotstudie zur Anwendbarkeit des Programms AKTIVA bei türkischen Migranten/innen. Diese ersten Ergebnisse sprechen für Wirksamkeit des Programms in Hinblick auf einen Anstieg der kognitiven Leistungsfähigkeit. Bedingungen für eine erfolgreiche Durchführung werden diskutiert.

Mag. Dr. Olivia Kada

\section{Korrespondenzadresse}

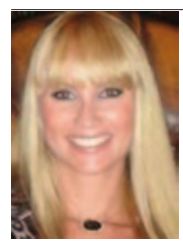

Mag. Dr. O. Kada

Studiengang Gesundheitsund Pflegemanagement

Fachhochschule Kärnten Hauptplatz 12

9560 Feldkirchen in Kärnten o.kada@fh-kaernten.at

\section{Literatur}

1. Antonovsky A (1985) Health, stress and coping. Jossey Bass, San Francisco

2. Antonovsky A (1996) The salutogenetic model as a theory to guide health promotion. Health Promot Int 11(1):11-18

3. Kickbusch I (1996) Tribute to Aaron Antonovsky ,What creates health'. Health Promot Int 11(1):5-6

4. Singer S, Brähler E (2007) Die „Sense of Coherence Scale". Testhandbuch zur deutschen Version. Vandenhoeck \& Ruprecht, Göttingen 